\title{
Improving Robot Navigation in Structured Outdoor Environments by Identifying Vegetation from Laser Data
}

\author{
Kai M. Wurm
}

Cyrill Stachniss

\author{
Wolfram Burgard
}

\begin{abstract}
This paper addresses the problem of vegetation detection from laser measurements. The ability to detect vegetation is important for robots operating outdoors, since it enables a robot to navigate more efficiently and safely in such environments. In this paper, we propose a novel approach for detecting low, grass-like vegetation using laser remission values. In our algorithm, the laser remission is modeled as a function of distance, incidence angle, and material. We classify surface terrain based on 3D scans of the surroundings of the robot. The model is learned in a self-supervised way using vibrationbased terrain classification. In all real world experiments we carried out, our approach yields a classification accuracy of over $99 \%$. We furthermore illustrate how the learned classifier can improve the autonomous navigation capabilities of mobile robots.
\end{abstract}

\section{INTRODUCTION}

Autonomous outdoor navigation is an active research field in robotics. In most of the outdoor navigation scenarios including autonomous cars, autonomous wheelchairs, surveillance robots, or transportation vehicles, the classification of the terrain plays an important role as most of the robots have been designed for navigation on streets or paved paths rather than on natural surfaces covered by grass or vegetation. The navigation outside of paved paths might be uncomfortable for passengers and might even introduce the risk of the robot getting stuck. Furthermore, driving on grass will in general increase wheel slippage and in this way increase potential errors in the odometry. Accordingly, the robust detection of vegetated areas is an important requirement for robots in any of the above-mentioned situations.

In this paper, we propose a novel laser-based classification approach that is especially suited for detecting low vegetation typically found in structured outdoor environments such as parks or campus sites. We classify surface terrain based on 3D scans of the surrounding of the robot in order to allow the robot to take the classification result into account during trajectory planning. It exploits an effect that is well known from satellite image analysis: Chlorophyll which is found in living plants strongly reflects near-IR light [13]. Often used laser scanners such as the SICK LMS 291-S05 scanner emit near-IR light and return the reflectivity of the object they hit. Our approach models this remission value of the laser scanner as a function of terrain class, incidence angle, and measured distance. Classification is done using a support

All authors are with the University of Freiburg, Department of Computer Science, D-79110 Freiburg, Germany

This work has partly been supported by the German Research Foundation (DFG) under contract number SFB/TR-8 (A3) and by the EC under contract number FP7-231888-EUROPA.

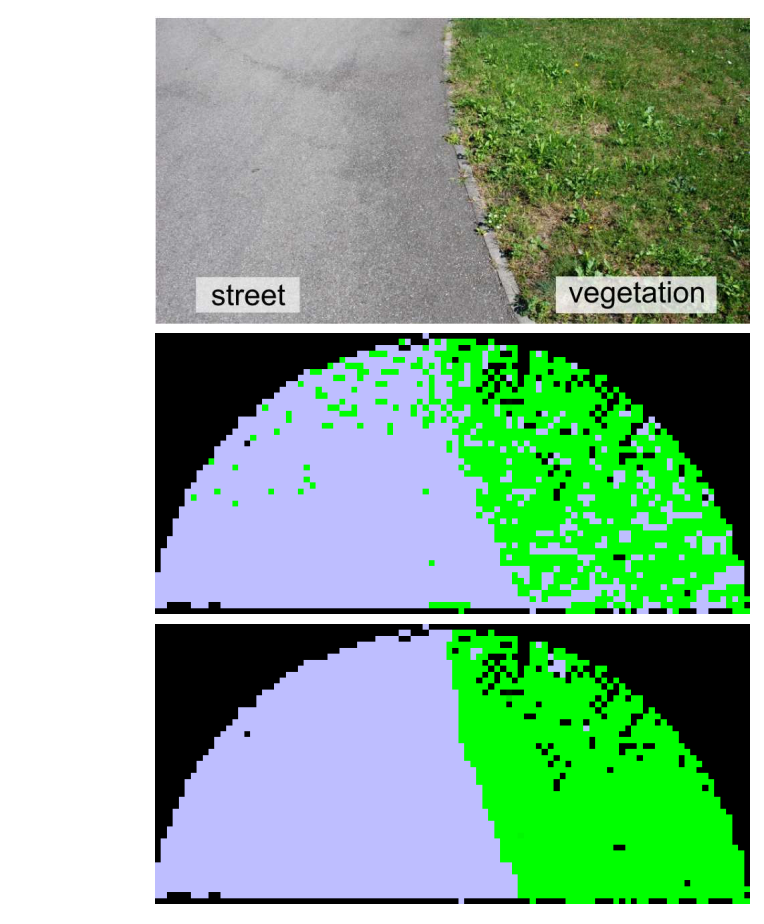

Fig. 1. Picture of a street and vegetation (top) and typical classification results obtained based on range differences (middle) and remission values (bottom). Shown is a bird's eye view of a 3D scan of the area depicted at the top with a maximum range of $5 \mathrm{~m}$. Whereas points classified as street are depicted in blue, points corresponding to vegetation are colored in green.

vector machine. To integrate classification results, we apply a probabilistic mapping method similar to occupancy grid mapping [12]. The model is learned in a self-supervised way using a vibration-based classification approach to label training data. In our experiments, we demonstrate that our approach can be used to accurately map vegetated areas and do so with a higher accuracy than standard techniques that are solely based on range values (see Fig. 1). We furthermore present an application to autonomous navigation in structured outdoor environments in which a robot benefits from the knowledge about the vegetation in its surroundings.

This paper is organized as follows. After discussing related work, we will briefly describe Support Vector machines which are employed for classification in our approach. Sec. IV then discusses the properties of the remission values. In Sec. V we then present our approach for self-supervised learning of the terrain classification. Finally, in Sec. VI we describe the experimental results obtained with real data and with real robots navigating through our university campus. 


\section{RELATED WORK}

There exist several approaches for detecting vegetation using laser measurements. Wolf et al. [23] use hidden Markov models to classify scans from a tilted laser scanner into navigable (e.g., street) and non-navigable (e.g., grass) regions. The main feature for classification is the variance in height measurements relative to the robot height. Other approaches analyze the distribution of $3 \mathrm{D}$ endpoints in a sequence of scans [8], [9], [10]. However, flat vegetation such as a freshly mowed lawn can not be reliably detected using this feature alone.

A combination of camera and laser measurements has been used to detect vegetation in several approaches [2], [6], [11], [22]. In a combined system, Wellington et al. [22] use the remission value of a laser scanner in addition to density features and camera images as a classification feature. However, they do not model the dependency between remission, measured range, and incidence angle. Probably due to this fact, they found the feature to be only "moderately informative".

The approach that is closest to our approach has been proposed by Bradley et al. [2]. Chlorophyll-rich vegetation is detected using a combination of laser range measurements, regular and near-infrared cameras. Vegetation is recognized by comparing measurements from the different types of cameras. 3D laser measurements of the environment are projected into the camera images. A classifier is then trained using the vegetation feature and features from the distribution of $3 \mathrm{D}$ endpoints. According to the authors the approach yields a classification accuracy of up to $95 \%$ but requires sophisticated camera equipment.

In contrast to those combined systems, our approach uses a laser scanner as its sole sensor. It is thus independent of lighting conditions and can be used on a variety of existing robot systems. Additionally, hand-labeling of training data is not required in our approach.

Terrain types have also been classified using vibration sensors on a robot [3], [7], [15], [21]. In these approaches, the robot traverses the terrain and the induced vibration is measured using accelerometers. The measurements are usually analyzed in the Fourier domain. Sadhukhan et al. [15] presented an approach based on neural networks. A similar approach is presented by DuPont et al. [7]. Brooks and Iagenemma [3] use a combination of principal component analysis and linear discriminant analysis to classify terrain. More recently, SVMs have been used by Weiss et al. [20], [21].

Self-supervised learning has previously been used by Dahlkamp et al. [5] in a vision-based road detection system. Here, laser measurements are used to identify nearby traversable surfaces. This information is then used to label camera image patches in order to train a classifier that is able to predict traversability in the far range. In our approach, we adopt the idea of self-supervision to generate labeled training data. We apply a vibration-based classifier to label laser measurements recorded by the robot. This labeled dataset is then used to train a laser-based vegetation classifier. Both classifiers used in our approach have been implemented using support vector machines which will be introduced in the following.

\section{Support Vector Machines}

Support vector machines (SVMs) are a kernel-based learning method which is widely used for classification and regression [16]. A SVM is essentially a hyperplane learning algorithm. Two classes of data points are separated by a hyperplane so that the margin between training points and the plane is maximized:

$$
\max _{w \in H, b \in \mathbb{R}} \min \left\{\left\|x-x_{i}\right\| \mid x \in H,\langle w, x\rangle+b=0\right\},
$$

where $H$ is some dot product space, $x_{i}$ are training points, and $w$ is a weighting vector. The following decision function is used to determine the class label $y$ :

$$
\begin{aligned}
f(x) & =\langle w, x\rangle+b \\
y & =\operatorname{sgn}(f(x))
\end{aligned}
$$

The hyperplane can be constructed efficiently by solving a quadratic programming problem. To separate non-linear classes, the so-called kernel trick is applied. The training data is first mapped into a higher-dimensional feature space using a kernel function. A separating hyperplane is then constructed in this features space which yields a nonlinear decision boundary in the input space. In practice, the Gaussian Radial Basis Function (RBF) is often used as a kernel function given by

$$
k\left(x, x^{\prime}\right)=e^{\frac{-\left(x-x^{\prime}\right)^{2}}{2 l^{2}}},
$$

with the so-called length-scale parameter $l$.

There exist derivatives of the basic SVM-formulation which allow for training errors. Among those, $C-\mathrm{SVM}$ is a popular method. An addition parameter commonly denoted as $C$ has to be optimized which adjusts the trade-off between maximizing the margin and minimizing the training error.

Throughout this work, we use the SVM implementation of LibSVM [4].

\section{USING REMISSION VALUES FOR TERRAIN ClasSIFICATION}

The goal of our terrain classification algorithm is to precisely classify the area containing vegetation. This classification has to be made early enough for the robot's planner to take the classification into account. For this reason, we focus on classifying three-dimensional scans of the environment surrounding the robot.

We are interested in distinguishing flat vegetation such as grass from drivable surfaces such as streets or paved paths. For sake of brevity, we will call those classes of materials "street" and "vegetation" in the following.

Compared to an approach based purely on the scan point distribution (see experiment in Sec. VI-A) a far better classification accuracy can be achieved when the remission value of laser measurements is used to classify endpoints. 


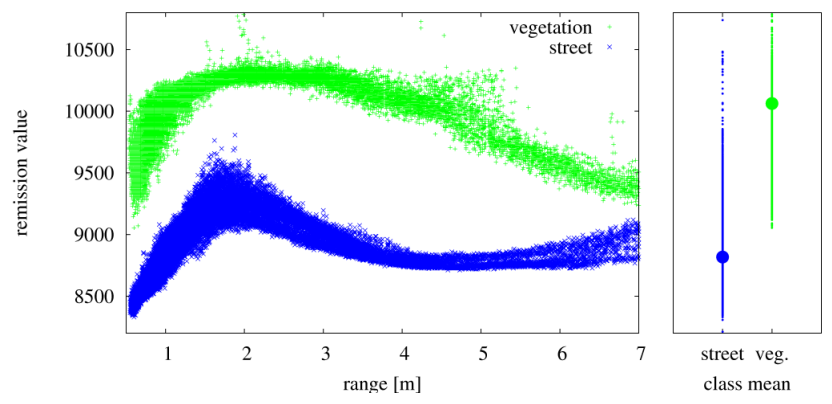

Fig. 2. Left: typical remission measurements of a SICK LMS 291-S05 for street (blue) and vegetation class (green). Right: mean remission values for both classes.

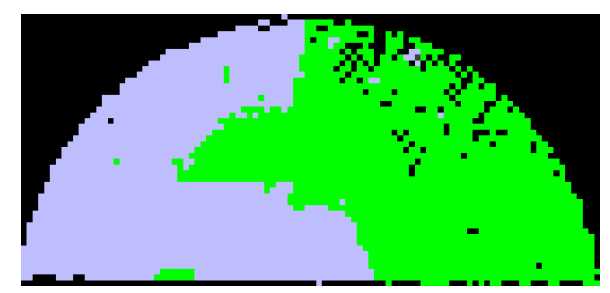

Fig. 3. Classification of the scene depicted in Fig. 1 using the mean remission value (see Fig. 2, right) to distinguish both classes.

The SICK LMS laser scanner uses light at a wavelength of $905 \mathrm{~nm}$ which is within the near-infrared range. Chlorophyll which is found in living plants strongly reflects near-IR light [13]. The remission values returned by the scanner depends on the material of the measured surface, on the distance at which it is hit, and on the angle of incidence [1]. Unfortunately, this relation is non-linear as the example of measurements in Fig. 2 shows. It is essential to take the measured range as well as the angle of the measurement into account. Averaging the remission value over all measured ranges and angles will lead to wrong classification results especially in the near range of up to three meters. This can be seen in Fig. 3. Here, the scene from Fig. 1 was classified using the mean remission value for both classes (see Fig. 2) ignoring the range and incidence angle.

In our approach, we apply a $C$-SVM to classify laser beams depending on the measured range, incidence angle, and remission using a RBF-kernel. Other classification methods might certainly be used instead of a SVM as long as they are able to classify data which is only separable in a non-linear way. The input to the learning algorithm is a labeled set of laser measurements. Each data point consists of a range measurement, incidence angle, and a remission value. By determining the separating hyperplane between both data sets, we implicitly model the remission functions for the street and vegetation class.

\section{SELF-SUPERVISED LEARNING FOR ROBUST TERRAIN ClassiFICATION}

To avoid tedious hand-labeling of dense three-dimensional training data our algorithm uses a self-supervised training method [5]. While the robot is moving it generates a 3D model of the environment using the method described in Sec. V-B. Incidence angles are estimated using the surface normal in the 3D model as well as the angle of the laser beam relative to the robot.

For each measured scan point the remission value, incidence angle, and distance are stored. As the robot moves through the environment it traverses previously scanned surface patches. Those patches are labeled using the vibration classifier described below and are then fed to the SVM as training data. The training is done offline. To optimize the length-scale parameter $l$ of the kernel as well as the softmargin parameter $C$, we perform a systematic grid search on the grid $\log _{2} l \in\{-15, \ldots, 3\}$ and $\log _{2} C \in\{-5, \ldots, 15\}$. The optimal parameters are determined using 5 -fold cross validation.

Once the classifier has been trained, the model can be used on any robot which is using the same sensor in a similar configuration. More specifically, the laser should be mounted so that the laser is measuring the surface at angles and distances similar to those observed during the training phase.

\section{A. Terrain Classification based on the Vibration of the Vehicle}

We use a vibration-based classifier to label training data. Different types of terrain induce vibrations of a different characteristic to the robot. These vibrations can be measured by an inertial measurement unit (IMU) and can be used to classify the terrain the robot traverses. Note that such classifiers do not allow the prediction of terrain classes in areas which have not been traversed yet. In our system, we only need to differentiate between vegetation and streets (non-vegetation) to generate training data for the SVM. In our experiments, we use a XSens MTi to measure the acceleration along the z-axis. We apply the Fourier transform to the raw acceleration data. In our algorithm, a 128-point FFT is used to capture the frequency spectrum of up to $25 \mathrm{~Hz}$.

The frequency spectrum also depends on the speed of the robot. To account for this dependency, it has been suggested to train several classifiers at different speeds [20]. In our system, however, we decided to treat the forward velocity as a training feature instead. In addition to this, we also use the rotational velocity as a feature to account for vibrations which result from the skid-steering of our robot.

To classify the acceleration and velocity data, we again use a $C$-SVM with a RBF-kernel. Our feature vector $x$ consists of the first 32 Fourier magnitudes $\left|F_{m}\right|$, the mean forward velocity $\bar{v}_{t}$ and the mean rotational velocity $\bar{v}_{r}$ of the robot over the sample period

$$
\begin{aligned}
\left|F_{m}\right| & =\sqrt{\left(\operatorname{Re} F_{m}\right)^{2}+\left(\operatorname{Im} F_{m}\right)^{2}}, m \in\{0, \ldots, 31\} \\
x & =\left\{\left|F_{0}\right|, \ldots,\left|F_{31}\right|, \bar{v}_{t}, \bar{r}_{t}\right\}
\end{aligned}
$$

where $F_{m}$ denotes the m-th Fourier component. The classifier was trained by recording short tracks of about $50 \mathrm{~m}$ at varying speeds both on a street and on vegetation. Parameter optimization was done using grid search and 5-fold cross validation. 
In our experiments, we achieved a high classification accuracy and thus this data is well suited to label the training data for the laser-based classification.

\section{B. Mapping of Vegetation}

We use multi-level surface maps [18] to model the environment. This representation uses a $2 \mathrm{D}$ grid and stores in each cell a set of patches representing individual surfaces at different heights. In our approach, we additionally store the probability of each surface patch to contain vegetation. Let $P\left(\mathrm{v}^{i}\right)$ denote this probability of patch $i$. In general, surface patches will be observed multiple times. Therefore, we need to probabilistically combine results from several measurements. In this way, the uncertainty in classification is explicitly taken into account.

Let $z_{t}$ be a laser measurement at time $t$. Analogous to Moravec [12], we obtain an update rule for $P\left(\mathrm{v}^{i} \mid z_{1: t}\right)$. First we apply Bayes' rule and obtain

$$
P\left(\mathrm{v}^{i} \mid z_{1: t}\right)=\frac{P\left(z_{t} \mid \mathrm{v}^{i}, z_{1: t-1}\right) P\left(\mathrm{v}^{i} \mid z_{1: t-1}\right)}{P\left(z_{t} \mid z_{1: t-1}\right)} .
$$

We then compute the ratio

$$
\frac{P\left(\mathrm{v}^{i} \mid z_{1: t}\right)}{P\left(\neg \mathrm{v}^{i} \mid z_{1: t}\right)}=\frac{P\left(z_{t} \mid \mathrm{v}^{i}, z_{1: t-1}\right)}{P\left(z_{t} \mid \neg \mathrm{v}^{i}, z_{1: t-1}\right)} \frac{P\left(\mathrm{v}^{i} \mid z_{1: t-1}\right)}{P\left(\neg \mathrm{v}^{i} \mid z_{1: t-1}\right)} .
$$

Similarly, we obtain

$$
\frac{P\left(\mathrm{v}^{i} \mid z_{t}\right)}{P\left(\neg \mathrm{v}^{i} \mid z_{t}\right)}=\frac{P\left(z_{t} \mid \mathrm{v}^{i}\right)}{P\left(z_{t} \mid \neg \mathrm{v}^{i}\right)} \frac{P\left(\mathrm{v}^{i}\right)}{P\left(\neg \mathrm{v}^{i}\right)},
$$

which can be transformed to

$$
\frac{P\left(z_{t} \mid \mathrm{v}^{i}\right)}{P\left(z_{t} \mid \neg \mathrm{v}^{i}\right)}=\frac{P\left(\mathrm{v}^{i} \mid z_{t}\right)}{P\left(\neg \mathrm{v}^{i} \mid z_{t}\right)} \frac{P\left(\neg \mathrm{v}^{i}\right)}{P\left(\mathrm{v}^{i}\right)} .
$$

If we apply the Markov assumption that the current observation is independent of previous observations given we know that a patch contains vegetation

$$
P\left(z_{t} \mid \mathrm{v}^{i}, z_{1: t-1}\right)=P\left(z_{t} \mid \mathrm{v}^{i}\right)
$$

and utilize the fact that $P\left(\neg \mathrm{v}^{i}\right)=1-P\left(\mathrm{v}^{i}\right)$, we obtain

$$
\begin{aligned}
& \frac{P\left(\mathrm{v}^{i} \mid z_{1: t}\right)}{1-P\left(\mathrm{v}^{i} \mid z_{1: t}\right)}= \\
& \quad \frac{P\left(\mathrm{v}^{i} \mid z_{t}\right)}{1-P\left(\mathrm{v}^{i} \mid z_{t}\right)} \frac{P\left(\mathrm{v}^{i} \mid z_{1: t-1}\right)}{1-P\left(\mathrm{v}^{i} \mid z_{1: t-1}\right)} \frac{1-P\left(\mathrm{v}^{i}\right)}{P\left(\mathrm{v}^{i}\right)} .
\end{aligned}
$$

This equation can be transformed into the following update formula:

$$
\begin{aligned}
& P\left(\mathrm{v}^{i} \mid z_{1: t}\right)= \\
& \quad\left[1+\frac{1-P\left(\mathrm{v}^{i} \mid z_{t}\right)}{P\left(\mathrm{v}^{i} \mid z_{t}\right)} \frac{1-P\left(\mathrm{v}^{i} \mid z_{1: t-1}\right)}{P\left(\mathrm{v}^{i} \mid z_{1: t-1}\right)} \frac{P\left(\mathrm{v}^{i}\right)}{1-P\left(\mathrm{v}^{i}\right)}\right]^{-1}
\end{aligned}
$$

To perform the update step, we need an inverse sensor model $P\left(\mathrm{v}^{i} \mid z\right)$. In our system, this sensor model is based on the remission value of the laser. The SVM-classifier is used to model the measurement probabilities as described in the next section. Here, the prior probability of $P\left(\mathrm{v}^{i}\right)$ was set to 0.5 .

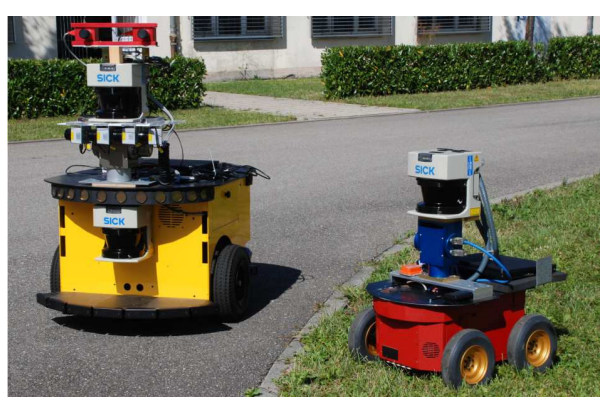

Fig. 4. Robots used in our experiments.

\section{Class Probabilities}

The decision function of Support Vector Machines (see Eq. 3) produces a class label $y$ which is not a probability ( $y$ is either 1 or -1 ).

Several methods have been proposed to map the output of SVMs to posterior class probabilities [14], [19]. Among those a popular approach is Platt's method [14]. It uses a parametric model to fit the posterior given by

$$
P(y=1 \mid f)=(1+\exp (A f+B))^{-1} .
$$

The parameters $A$ and $B$ are determined using maximum likelihood estimation from a training set $\left(f_{i}, y_{i}\right)$, where $f_{i}$ are the outputs of the function given in Eq. 2 and $y_{i}$ are the corresponding class labels. For details of the optimization step see [14]. In our approach, this method is used to obtain the inverse sensor model $P\left(\mathrm{v}^{i} \mid z\right)$.

\section{EXPERIMENTS}

Our approach has been implemented and evaluated in several experiments. The experiments are designed to demonstrate that our approach is suitable to allow robots to reliably detect vegetation and thus improves robust navigation in structured outdoor environments.

We used two different robot systems (see Fig. 4). The selfsupervised learning approach is evaluated using an ActivMedia Pioneer 2 AT, which is able to traverse low vegetation. For mapping large environments and for an autonomous driving experiment, we use an ActivMedia Powerbot platform. This robot cannot safely traverse grass since its castor wheels will block the robot due to its weight. Both robots are equipped with SICK LMS S291-S05 laser scanners on pan-tilt units. In addition to that, the Pioneer robot carries an XSens MTi IMU to measure vibrations. Three-dimensional scans are gathered by tilting the laser scanner from 50 degrees upwards to 30 degrees downwards. We use the raw (unnormalized) remission values provided by the scanner.

We limit the classification to scans with a range smaller than 5.0 meters. The approach itself is not limited in range. However, at a laser resolution of one degree and a low height of the sensor relative to the ground (approx. $0.5 \mathrm{~m}$ ), long range data will be too sparse both to gather training data and to reliably detect drivable surfaces and obstacles.

\section{A. Vegetation detection using range differences}

In a first experiment, we implemented a vegetation detection algorithm based purely on the range differences of 


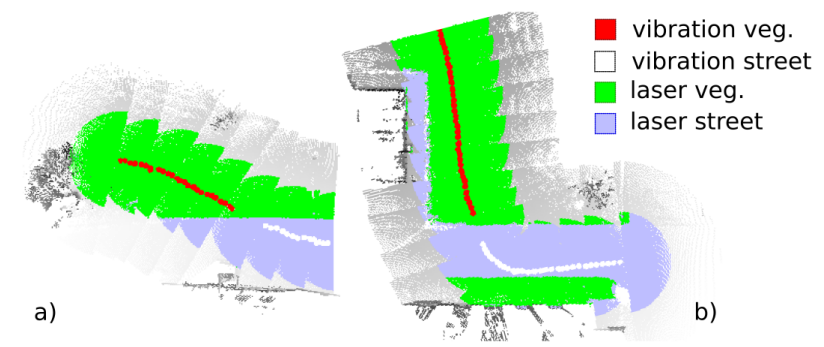

Fig. 5. Self-supervised learning. Left: the data set which is used to train the classifier. Right: test set recorded at a different location.

neighboring laser measurements similar to the one proposed by Wolf et al. [23]. This method is only used for a comparison with our proposed classification approach. Threedimensional data is acquired by gathering a sweep of 2D scans. For each $2 \mathrm{D}$ scan the $2 \mathrm{D}$ endpoints $p_{i}=\left\langle x_{i}, y_{i}\right\rangle$ are computed from the range and angle measurements $\left\langle r_{i}, \alpha_{i}\right\rangle$. A local feature $d_{i}$ is then determined for every scan point

$$
d_{i}=x_{i}-x_{i-1} \text {. }
$$

This feature captures the local roughness of the terrain. To cope with flat but tilted surfaces we classify scans based on the absolute difference in $d_{i}$ between neighboring range measurements as suggested in [11]. Furthermore, we also use the measured range as a training feature to account for the varying data density from near to far scans. A SVM is used to train a classifier based on these features.

In our experiments, we achieve a classification accuracy of about $75 \%$ using the described method. An example of the classification results can be seen in Fig. 1. Similar results have been reported by other researchers [2].

\section{B. Self-supervised Learning}

To train our remission-based classifier, we manually steered the Pioneer AT robot through an outdoor environment consisting of a street and an area covered with grass. We acquired 3D scans approximately every $4 \mathrm{~m}$. While the robot was driving the IMU measured the vibration induced to the robot. To correct odometry errors of the robot, we employed a state-of-the-art SLAM approach [17] and 3D scanmatching. We trained our classifier using the self-supervised approach described in Sec. V. The training set is visualized in Fig. 5a. The data recorded at the border region between street and vegetation were ignored since the precise location of the border cannot be determined using the vibration sensor. The model for the laser-based classifier was trained using 19,989 vegetation and 11,248 street samples.

To further evaluate the precision of the classifier, we recorded separate test data at a different location (see Fig. 5b and Fig. 6). The test set contains 36,304 vegetation and 28,883 street measurements. Again, the labeling of the data was carried out using the vibration-based classifier. The previously trained classifier reached a precision of $99.9 \%$ on the test data; the recall is $99.6 \%$. The confusion matrix is given in Table I. Note that such accurate classification results are not due to overfitting. Fig. 2 illustrates that a non-linear function (as learned by the SVM in our approach) can clearly separate the classes.
TABLE I

CONFUSION MATRIX FOR EXP. VI-B (NUMBER OF DATA POINTS)

\begin{tabular}{|l|r|r|}
\hline & vegetation & street \\
\hline vegetation & 36,300 & 138 \\
\hline street & 4 & 28,745 \\
\hline
\end{tabular}

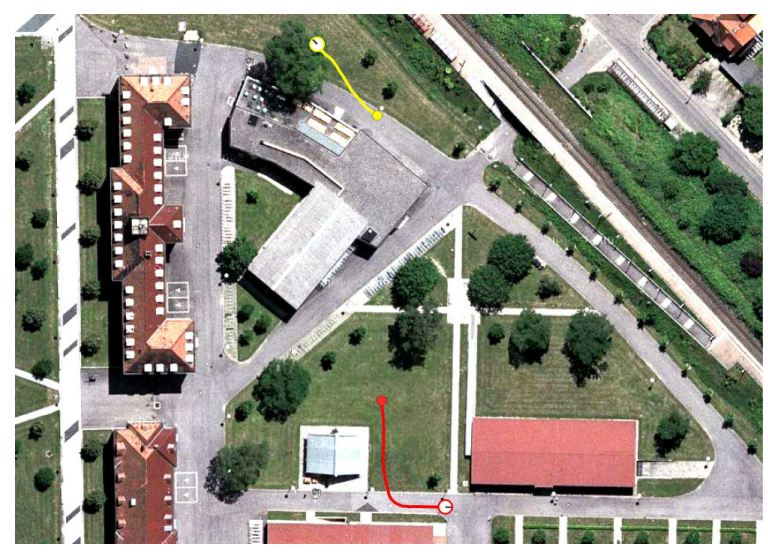

Fig. 6. Aerial view of the computer science campus in Freiburg. Approximated robot trajectories are shown for the training (top, yellow) and the test set (bottom, red). Courtesy of Google Maps, Copyright 2008, DigitalGlobe.

\section{3D Mapping}

In this experiment, the Powerbot robot was steered across the computer science campus at the University of Freiburg. The scanning laser of the robot was tilted to a fixed angle of 20 degrees downwards. In this way, a fairly large area could be mapped in less than 15 minutes. The length of the trajectory is $490 \mathrm{~m}$. The vegetation classifier was used to map vegetation in the three-dimensional model of the environment. To properly integrate multiple measurements, we used the mapping approach described in Sec. V-B with a cell size of $0.1 \mathrm{~m}$.

Due to a significantly different hardware setup than on the Pioneer AT, we were not able to use the model generated in Sec. VI-B. Instead, we recorded a training set of 12,153 grass and 10,448 street samples by placing the robot in front of flat areas containing only street and only vegetation. This method is only applicable if such example data can be gathered and thus should be considered inferior to the self-supervised approach described in Sec. V.

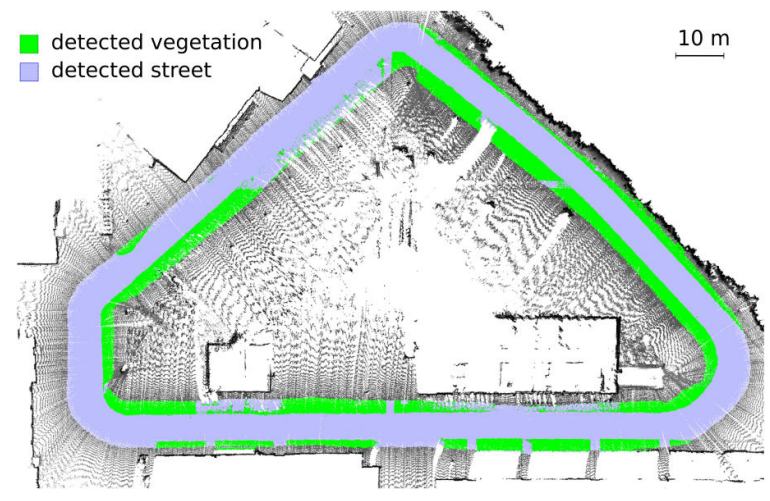

Fig. 7. Mapping of a large outdoor environment. The laser was tilted at a fixed angle of 20 degrees while the robot was moving. The figure shows a $2 \mathrm{D}$ projection of the $3 \mathrm{D}$ map. 


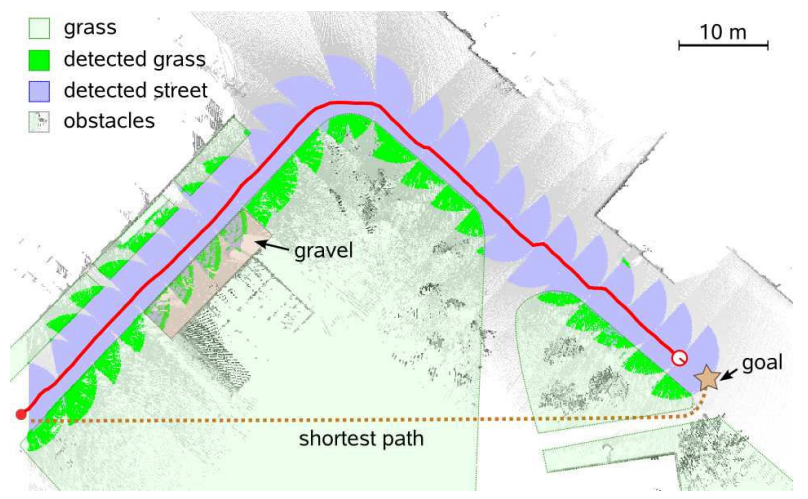

Fig. 8. Autonomous navigation experiment. Although the shortest obstaclefree path from the start to the goal position led over grass, the robot could reliably avoid the vegetated areas by using our vegetation classifier and traveled over the paved streets to reach its goal.

Compared to the aerial image of the campus site in Fig. 6, the mapping result shown in Fig. 7 is highly accurate. Even small amounts of vegetation, for example between tiles on a path, can be identified. To evaluate the accuracy of the map created during this experiment, we manually marked wrongly classified cells. Of a total of 271,638 cells (75,622 vegetation, 196,016 street), we found 547 false positives and 194 false negatives. This corresponds to a precision of $99.23 \%$ and a recall of $99.74 \%$.

\section{Autonomous Navigation}

As mentioned above, the Powerbot cannot safely traverse vegetated areas. In the experiment depicted in Fig. 8 (see also our video attachment), the robot was told to navigate to a goal position $80 \mathrm{~m}$ in front while avoiding vegetation. Since the robot did not have a map, it explored the environment in the process of reducing its distance to the goal location. Thereby, it used the vegetation classifier to detect vegetation. The environment was represented as described in Sec. V-B. Without knowledge about the specific terrain, the shortest obstacle-free path would have led the robot across a large area containing grass. By considering the classification results in the path costs, however, the planner chose a safe trajectory over the street.

\section{CONCLUSION}

In this paper, we proposed a new approach to vegetation detection using the remission values of a laser scanner. By predicting vegetation in the surrounding of a robot, our approach improves robot navigation in structured outdoor environments. The laser classifier is learned in a selfsupervised fashion by means of a support vector machine using a vibration-based terrain classifier to gather training data. The approach has been implemented and evaluated in several real-world experiments. The experiments show that our approach is able to accurately detect low, grasslike vegetation with an accuracy of more than $99 \%$. We also demonstrated that the terrain classification can be used to improve the navigation behavior of a robot.

Our current approach is limited to detecting flat vegetation due to the self-supervised training method. In future work, we will investigate whether the described approach can also be applied to classify tall vegetation such as trees or bushes. We will also look into using remission values provided by the recently introduced Hokuyo UTM-30LX. With a weight of $370 \mathrm{~g}$ this sensor could allow vegetation detection on an even broader range of robots including humanoids and small scale robots.

\section{REFERENCES}

[1] R. Baribeau, M. Rioux, and G. Godin. Color reflectance modeling using a polychromatic laser range sensor. Pattern Analysis and Machine Intelligence, IEEE Transactions on, 14(2):263-269, 1992.

[2] D. Bradley, R. Unnikrishnan, and J. Bagnell. Vegetation detection for driving in complex environments. In Proc. of the IEEE Int. Conf. on Robotics \& Automation (ICRA), 2007.

[3] C.A. Brooks, K. Iagnemma, and S. Dubowsky. Vibration-based terrain analysis for mobile robots. In Proc. of the IEEE Int. Conf. on Robotics \& Automation (ICRA), pages 3415-3420, 2005.

[4] C-C. Chang and C-J. Lin. LIBSVM: a library for support vector machines. http://www.csie.ntu.edu.tw/c̃jlin/libsvm, 2001.

[5] H. Dahlkamp, A. Kaehler, D. Stavens, S. Thrun, and G. Bradski. Selfsupervised monocular road detection in desert terrain. In Proc. of Robotics: Science and Systems (RSS), Philadelphia, USA, 2006.

[6] B. Douillard, D. Fox, and F. Ramos. Laser and vision based outdoor object mapping. In Proceedings of Robotics: Science and Systems IV, Zurich, Switzerland, 2008.

[7] E.M. DuPont, R.G. Roberts, C.A. Moore, M.F. Selekwa, and E.G. Collins. Online terrain classification for mobile robots. In Proc. of the Int. Mechanical Engineering Congress and Exposition Conference (IMECE), Orlando, USA, 2005.

[8] M. Hebert and N. Vandapel. Terrain classification techniques from ladar data for autonomous navigation. In Proc. of the Collaborative Technology Alliances conference, College Park, MD., 2003.

[9] J-F. Lalonde, N. Vandapel, D. Huber, and M. Hebert. Natural terrain classification using three-dimensional ladar data for ground robot mobility. Journal of Field Robotics, 23(10):839 - 861, 2006.

[10] J. Macedo, R. Manduchi, and L. Matthies. Ladar-based discrimination of grass from obstacles for autonomous navigation. In ISER 2000: Experimental Robotics VII, London, UK, 2001.

[11] R. Manduchi, A. Castano, A. Talukder, and L.Matthies. Obstacle detection and terrain classification for autonomous off-road navigation. Autonomous Robots, 18, pages 81-102, 2003.

[12] H.P. Moravec. Sensor fusion in certainty grids for mobile robots. AI Magazine, pages 61-74, 1988.

[13] R.B. Myneni, F.G. Hall, P.J. Sellers, and A.L. Marshak. The interpretation of spectral vegetation indexes. IEEE Transactions on Geoscience and Remote Sensing, 33(2):481-486, 1995.

[14] J.C. Platt. Probabilistic outputs for support vector machines and comparisons to regularized likelihood methods. In Advances in Large Margin Classifiers, pages 61-74, 1999.

[15] D. Sadhukhan, C. Moore, and E. Collins. Terrain estimation using internal sensors. In Proc. of the IASTED Int. Conf. on Robotics and Applications, Honolulu, Hawaii, USA, 2004.

[16] B Schölkopf and A. Smola. Learning with Kernels. MIT Press, 2002.

[17] C. Stachniss and G. Grisetti. GMapping project at OpenSLAM.org. http://openslam.org, 2007.

[18] R. Triebel, P. Pfaff, and W. Burgard. Multi-level surface maps for outdoor terrain mapping and loop closing. In Proc. of the IEEE/RSJ Int. Conf. on Intelligent Robots and Systems (IROS), 2006.

[19] V.N. Vapnik. Statistical Learning Theory. Wiley-Interscience, 1998.

[20] C. Weiss, N. Fechner, M. Stark, and A. Zell. Comparison of different approaches to vibration-based terrain classification. In Proc. of the European Conf. on Mobile Robots (ECMR), Freiburg, Germany, 2007.

[21] C. Weiss, H. Frohlich, and A. Zell. Vibration-based terrain classification using support vector machines. In Proc. of the IEEE/RSJ Int. Conf. on Intelligent Robots and Systems (IROS), 2006.

[22] C. Wellington, A. Courville, and A. Stentz. A generative model of terrain for autonomous navigation in vegetation. International Journal of Robotics Research, 25(12):1287 - 1304, 2006.

[23] D.F. Wolf, G. Sukhatme, D. Fox, and W. Burgard. Autonomous terrain mapping and classification using hidden markov models. In Proc. of the IEEE Int. Conf. on Robotics \& Automation (ICRA), 2005. 